\title{
A new case of fibrochondrogenesis from Spain
}

\author{
M L Martínez-Frías, A García, J Cuevas, J I Rodríguez, M Urioste
}

\begin{abstract}
A rare, neonatally lethal chondrodysplasia with histological characteristics was first described in 1978 and the authors named the condition fibrochondrogenesis. Here we report the eighth published case of fibrochondrogenesis. This was identified in a population of 1158067 consecutive livebirths, so we can assume that this figure should be the minimal prevalence for livebirths.
\end{abstract}

( $\mathcal{H}$ Med Genet 1996;33:429-431)

Key words: chondrodysplasia; skeletal dysplasia; fibrochondrogenesis.

Lazzaroni-Fossati et al described for the first time a rare, neonatally lethal chondrodysplasia named fibrochondrogenesis because of its histological characteristics. Since then, six more cases have been published..$^{2-4}$

We report a case with fibrochondrogenesis identified among the 1158067 livebirths registered by the Spanish Collaborative Study of Congenital Malformations (ECEMC).

\section{ECEMC and}

Departamento de

Farmacología.

Facultad de Medicina, Universidad Complutense, 28040 Madrid, Spain M L Martínez-Frías

Servicio de Pediatría, Residencia Sanitaria de la Seguridad

Social, Guadalajara,

Spain

A García

Servicio de Anatomía Patológica, Residencia Sanitaria de la

Seguridad Social,

Guadalajara, Spain

J Cuevas

Servicio de Anatomía Patológica, Hospital La Paz, Madrid, Spain

J I Rodriguez

ECEMC y Hospital Universitario San Carlos, Facultad de Medicina, Universidad Complutense, Madrid, Spain

M Urioste

Correspondence to: Dr Martínez-Frías.

Received 16 October 1995 Revised version accepted for publication 2 January 1996

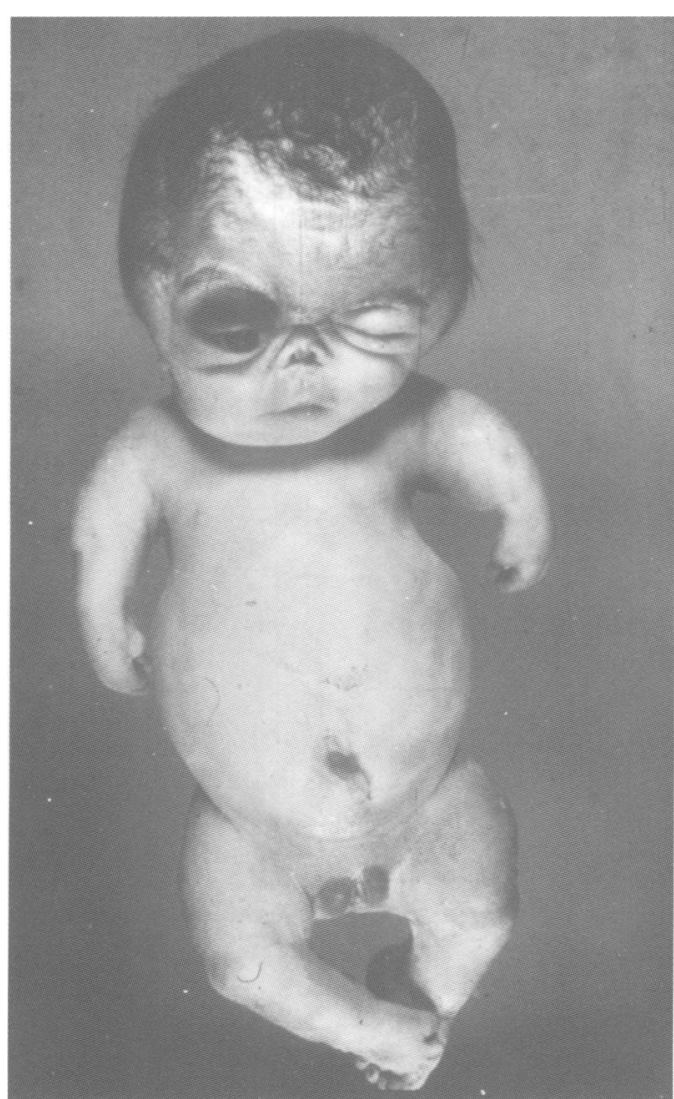

Figure 1 Clinical appearance of the patient.

\section{Case report}

The proband was the result of the first pregnancy of a 23 year old mother and 22 year old father. The parents, both from the same village although it has more than 22000 inhabitants, were healthy and denied consanguinity. The pregnancy was only complicated by an episode of flu during the first month that was treated with antibiotics, and by diarrhoea during the sixth month treated with antidiarrhoeal drugs. The infant, a girl weighing $2320 \mathrm{~g}(<3$ rd centile), was delivered by caesarean section at 40 weeks of gestation. Her length was $37 \mathrm{~cm}$ ( $<<3$ rd centile). Physical examination at birth showed relative macrocephaly with a widely patent fontanelle, protuberant eyes especially the right one owing to a periorbital haematoma (fig 1), flat nasal root with anteverted nares causing a particularly hypoplastic nose, short neck, narrow chest, and marked rhizomelic limb shortness. Cerebral ultrasound indicated dilated lateral ventricles. She lived for nine hours. Karyotyping was not performed.

Radiological examination showed deficient ossification of the skull bones, with evident frontal bossing (fig 2). The thorax was narrow and bell shaped with thick, short ribs. There was absence of some cervical and lumbar vertebral bodies (fig 3 ). The remaining vertebral bodies were flattened throughout the cervical,

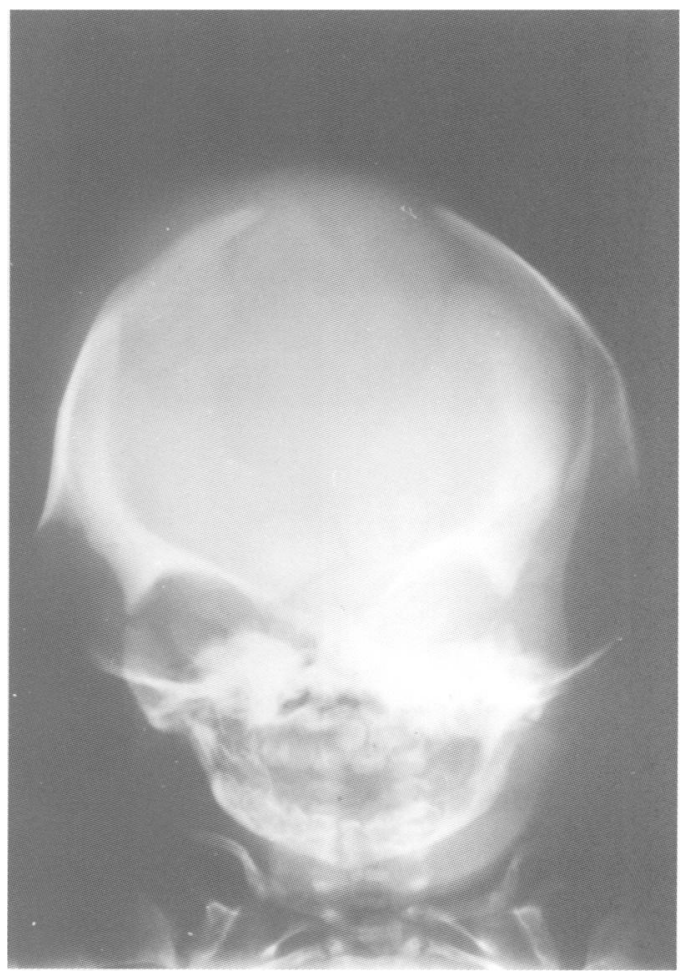

Figure $2 X$ ray of the head. Note the deficient ossification of the skull bones. 


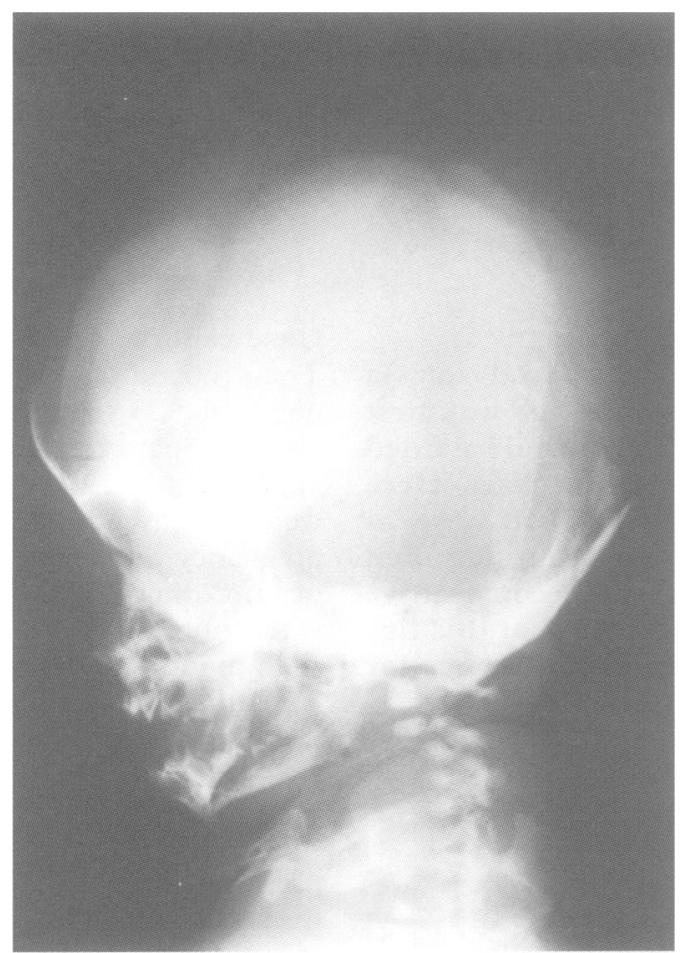

Figure 3 Lateral projection showing the absence of some cervical vertebral bodies.

thoracic, and lumbar spine (fig 4). On lateral projection (fig 5), the ossification was more extensive anteriorly giving a pear shaped appearance. The pelvis was hypoplastic with small ilia, which had small spurs, and poorly ossified

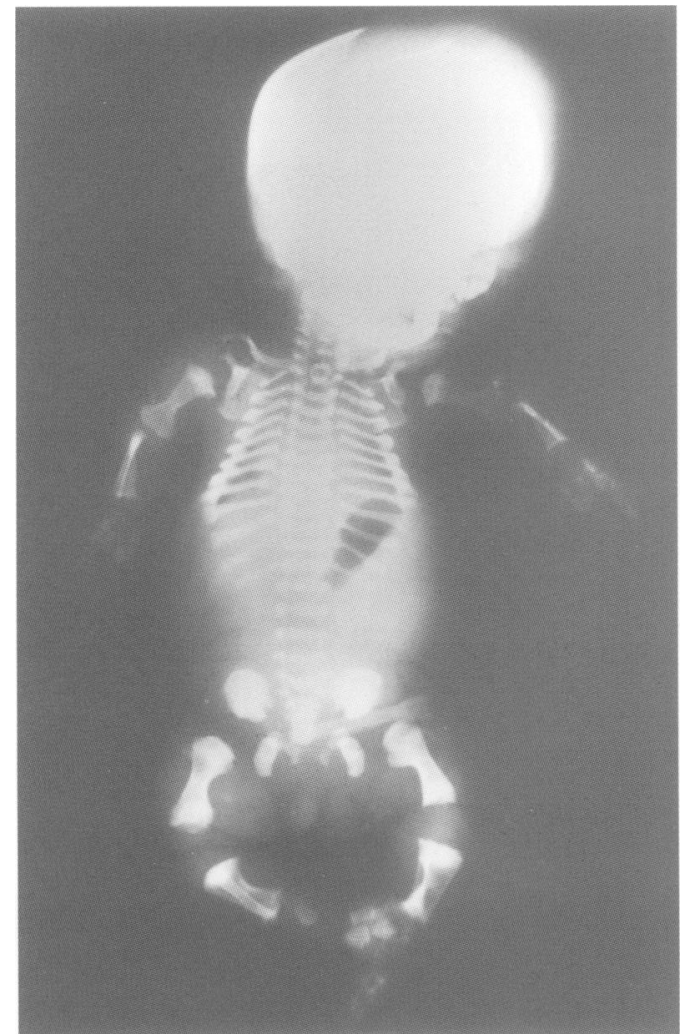

Figure $4 X$ ray of the patient. Note the flattened vertebral bodies and the shortness of the long tubular bones which werre broad. The iliac bones were small with spurs that were also present in the metaphyses of the humeri and femora.

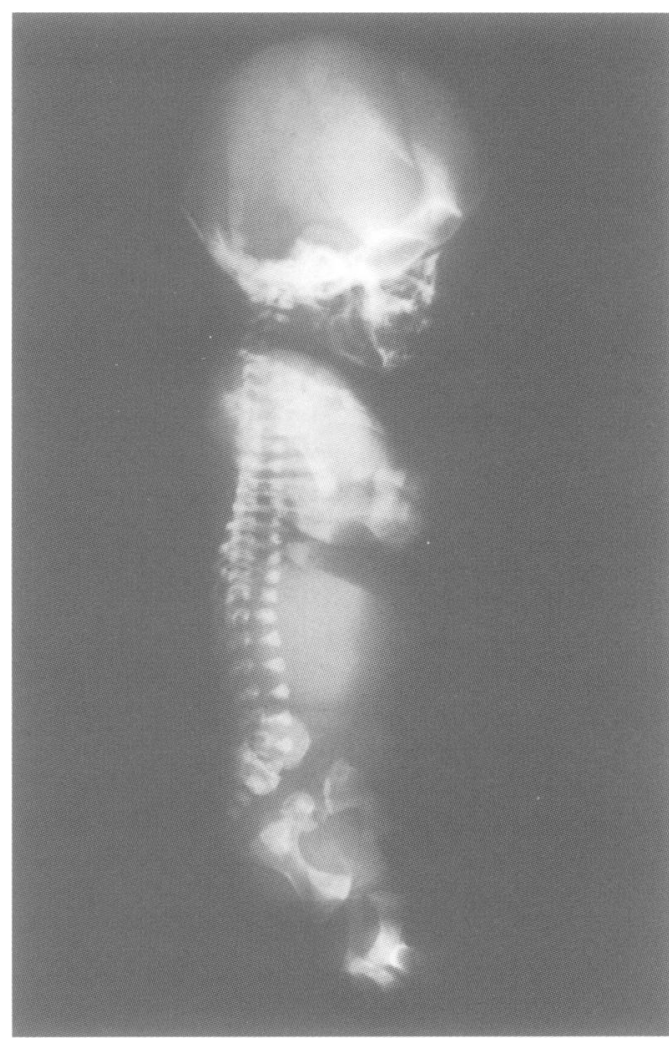

Figure 5 Lateral projection showing the pear shaped appearance of the vertebrae.

pubic rami. The long tubular bones were very short, bowed, and broad. The proximal and distal ends of the humeri and the femora were convex and also had metaphyseal spurs (fig 4). Necropsy showed normal internal organs. Microscopic examination of cartilage from the larynx and trachea showed findings similar to those for the rest of the bones in chondrocytes and cartilaginous matrix. Histological sections from different long bones showed striking abnormalities of chondrocytes and cartilaginous matrix in growth plates and epiphyseal cartilages. There was no demarcation between resting and proliferative zones of the epiphysis. Chondrocytes were rounded or, more often, spindle shaped with dense, hyperchromatic nuclei, and were arranged in clusters with intervening thick and fibrous septa of extracellular matrix (fig 6). Areas of cystic degeneration of the matrix cartilage were

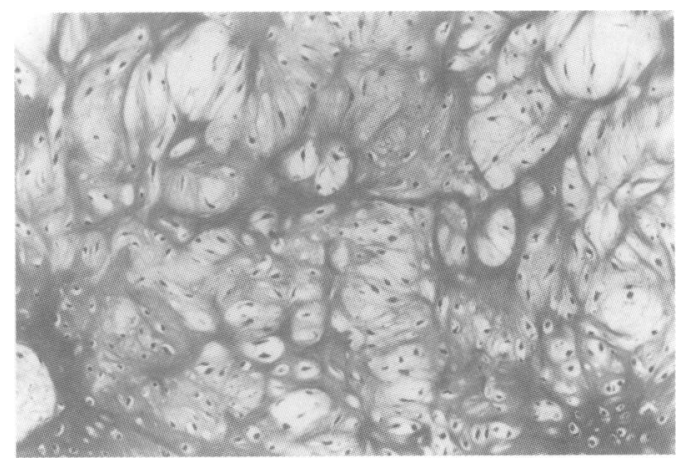

Figure 6 Epiphyseal cartilage showing abnormal groups of spindle shaped chondrocytes surrounded by fibrillar extramedular matrix. (Masson trichrome.) 


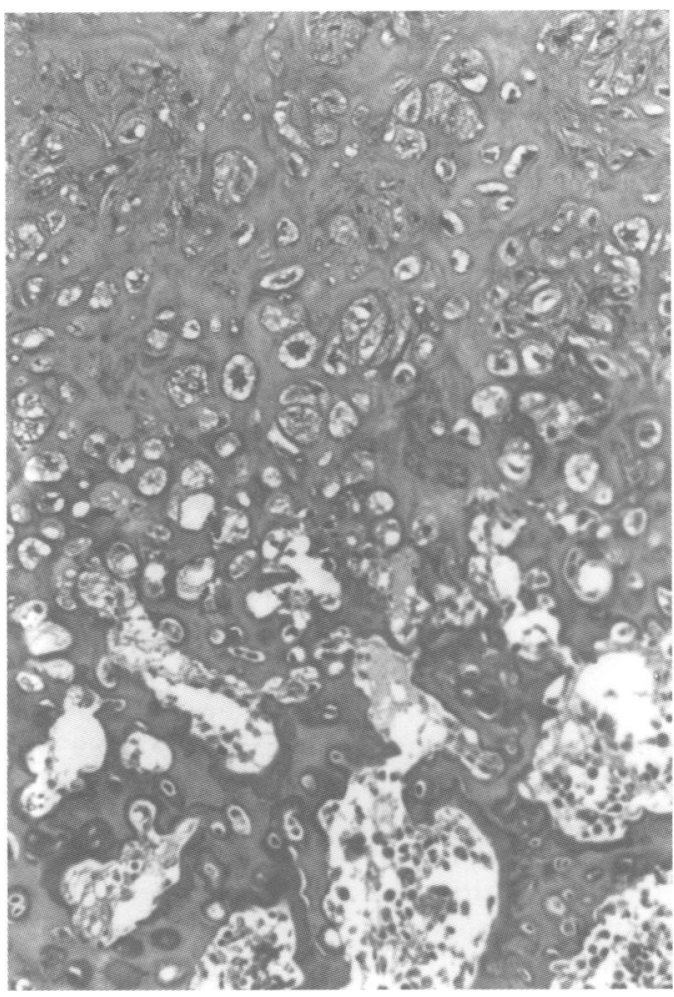

Figure 7 Long bone growth plates were disorganised. Note absence of columnisation of abnormal shape of hypertrophic cells, and thick metaphyseal bone trabeculae. (Masson trichrome.)

common. The growth plates showed no organisation and columnisation in normal proliferative zones. Cells in the plates were clustered in small nests with no formation of normal longitudinal septa (fig 7). Metaphyseal trabeculae were scarce, thick, and irregular.

\section{Discussion}

The clinical appearance of fibrochondrogenesis is quite similar to several of the lethal bone dysplasias. Thus, a clinical and radiological differential diagnosis is necessary, especially with achondrogenesis I and II, metatrophic dysplasia, thanatophoric dysplasia, atelosteogenesis type I, boomerang dysplasia, and schneckenbecken dysplasia. However, the clinical appearance in fibrochondrogenesis is quite different, especially the face with protuberant eyes and an unusual, hypoplastic nose that appears to be distinctive. The facial appearance of our case is markedly similar to the cases published by Eteson et al, Whitely et $a l,{ }^{3}$ and Bankier $e t a l,{ }^{4}$ and the radiological features are sufficiently distinctive to suspect the diagnosis of fibrochondrogenesis. Nevertheless, the final diagnosis is made on histological findings, specifically the fibrosis of the growth plate cartilage.

The case we present here is the eighth published case. As this infant was identified in a population of consecutive livebirths, we can assume that the prevalence is 1 per 1158067 livebirths. Nonetheless, this figure should be considered a minimum, since we cannot totally exclude that some cases with lethal forms of bone dysplasias, who died without radiological or pathological studies, were not identified as having fibrochondrogenesis.

The condition has been considered to be autosomal recessive on the basis that the case described by Lazzaroni-Fossati et $a l^{1}$ was the product of an uncle-niece marriage, who also had a previous sib with micromelia and cleft palate who died soon after birth, and on the concordance of male twins. ${ }^{4}$ However, all the other cases, including the present one, were sporadic and born to non-consanguineous parents. Thus, we consider that although it is probable that the condition is recessive, at present, other forms of inheritance cannot be totally excluded.

This work was supported, in part, by a Grant from Direccion General de Salud Pública (Ministerio de Sanidad y Consumo) of Spain, and by Fundacion 1000 of Spain.

1 Lazzaroni-Fossati F, Stanescu V, Stanescu R, Serra G, Magliano P, Maroteaux P. La fibrochondrogenese. Arch Fr Pediat 1978;35:1096-104

2 Eteson DJ, Adomian GE, Ornoy A, et al. Fibrochondrogenesis: radiologic and histologic studies. $A m \mathcal{F}$ Med Genet 1984;19:277-90.

3 Whitley CB, Langer LO Jr, Ophoven J, et al. Fibrochondrogenesis: lethal, autosomal recessive chondrodysplasia with distinctive cartilage histopathology. $\mathrm{Am}$ drodysplasia with distinctive $1984 ; 19: 265-75$.

4 Bankier A, Fortune D, Duke J, Sillence DO. Fibrochondrogenesis in male twins at 24 weeks gestation. Am F Med Genet 1991;38:95-8. 